\title{
High Resolution Eddy Current Tomography System for Nondestructive Testing
}

\author{
J. SALACH ${ }^{a}$, R. SZEWCZYK ${ }^{b, *}$
}

${ }^{a}$ Institute of Metrology and Biomedical Engineering, Warsaw University of Technology, Warsaw, Poland

${ }^{b}$ Industrial Research Institute for Automation and Measurements, Al. Jerozolimskie 202, 02-486 Warsaw, Poland

Idea of the high-resolution eddy current tomography is presented. Proposed system gives possibility of testing cylinder-shaped elements made of both magnetic and non-magnetic materials. To validate the concept, the tomographic measurements are carried out on set of steel cylinders with non-magnetic copper inclusion. Measurements were done during both linear and rotational movement of the element. Achieved results indicate the high sensitivity of system, which creates possibility of its application for non-destructive testing of the elements made of ferromagnetic and non-magnetic materials.

DOI: $10.12693 /$ APhysPolA.126.402

PACS: 81.70.Ex, 81.70.Tx

\section{Introduction}

The tomography creates new possibilities for nondestructive testing technology. However, X-ray based tomography creates occupational safety problems [1]. For this reason application of X-ray for tomography is expensive, problematic and sometimes dangerous for operators.

The paper presents newly developed experimental setup for high resolution eddy current tomography. Presented solution enables the analysis of internal structure of tested sample made of conductive material [2], both magnetic as well as non-magnetic [3,4]. Presented eddy current tomography setup is safe for operators and can be easily adapted for industrial quality control systems [5]. As a result, such method of non-destructive testing is very attractive in recently developed quality control systems.

\section{Method of investigation}

The schematic block diagram of experimental setup is presented in Fig. 1, whereas Fig. 2 presents the model element used for tests.

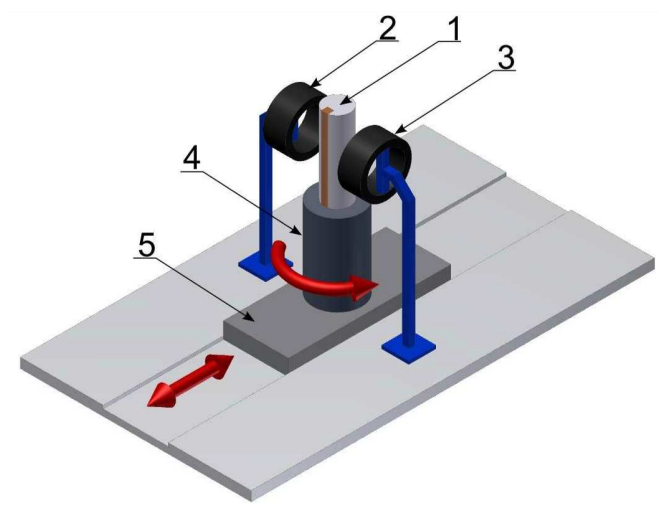

Fig. 1. Schematic block diagram of experimental setup for high resolution eddy current tomography: 1 - tested element, 2 - driving coil, 3 - detection coil, 4 - stepper motor for rotation, 5 - linear actuator.

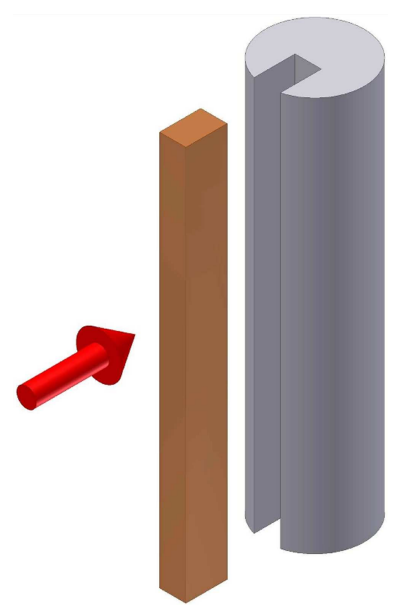

Fig. 2. Model element consisting of a construction steel ST3 cylinder with a copper inclusion.

Driving coil (2) is connected to sine wave current source. The measured signal from detection coil (3) contains both the amplitude and the phase shift. This signal is filtered by a band-pass filter to decrease noise level. On the basis of these data, internal structure of tested element (1), from the point of view of magnetic permeability and conductivity, may be calculated on the basis of Maxwell equations.

During the measurement, the test object is rotated by the stepper motor (4). Accordingly to the needs, object is moved by linear actuator (5).

\section{Results}

Experiment was carried out for three cylinders made of ST3 construction steel. In each cylinder a copper block inclusion was made. The inclusion block was inserted $13 \mathrm{~mm}$ into the cylinder, whereas its width was $4 \mathrm{~mm}$, $8 \mathrm{~mm}$ and $12 \mathrm{~mm}$ respectively. As it is presented in Fig. 2, the copper block inclusion was made along the entire length of the model element. 


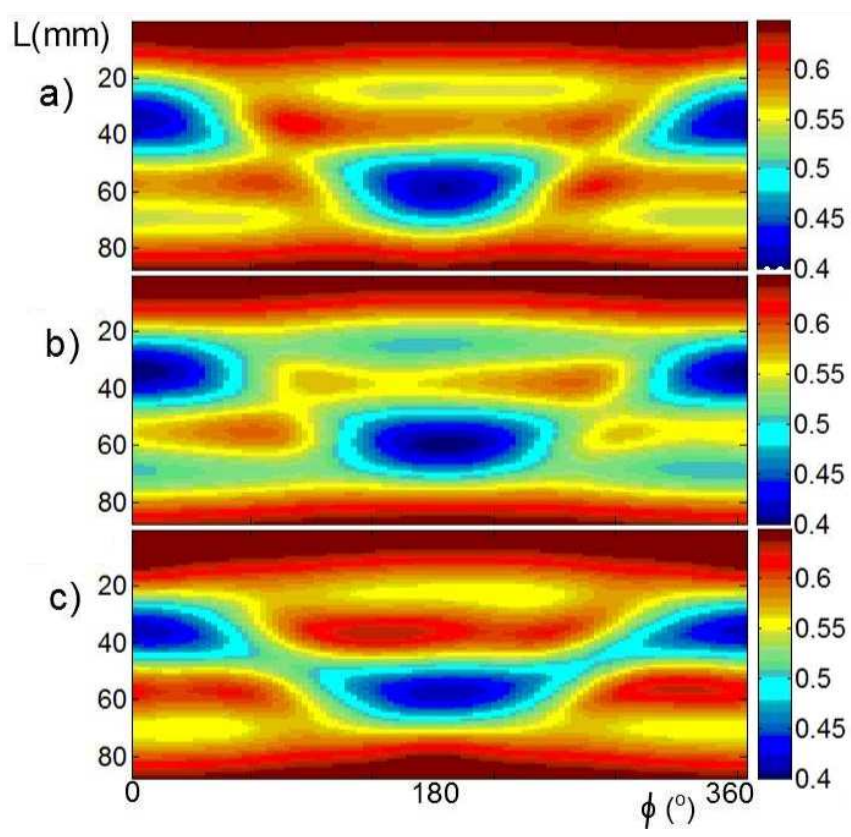

Fig. 3. Visualized results of measurements the value of amplitude of the signal on detection coil as a function of linear movement $L$ and rotation $\varphi$ for model steel elements with copper inclusions width: a) $4 \mathrm{~mm}$, b) $8 \mathrm{~mm}$, c) $12 \mathrm{~mm}$.

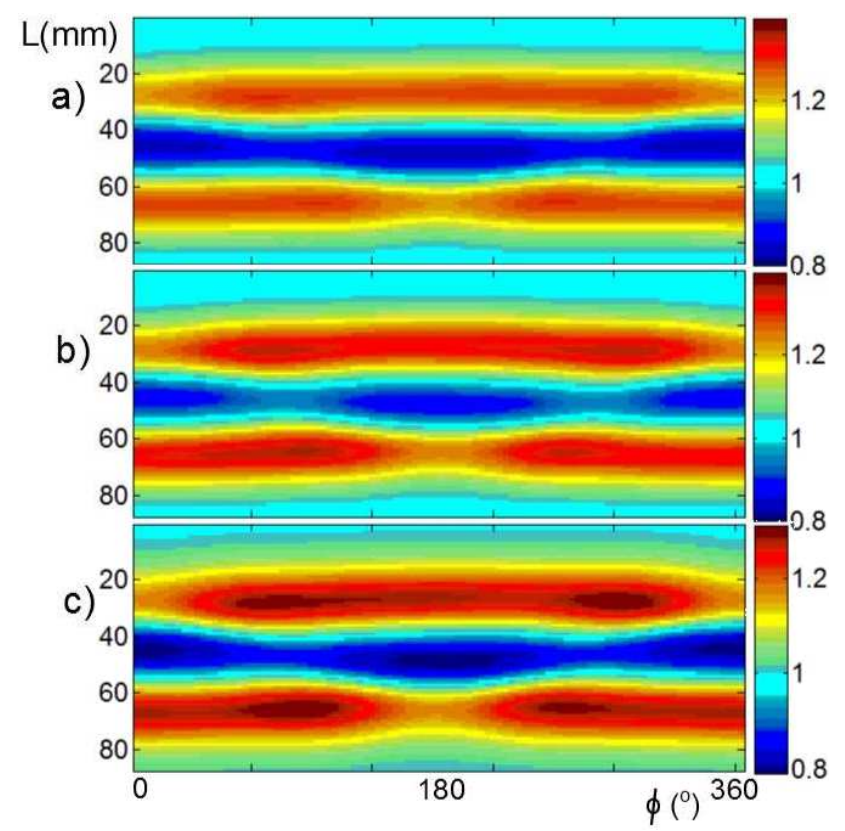

Fig. 4. As Fig. 3 for tangent of phase angle between signal on measuring coil and signal applied to driving coil.
The results of measurements of amplitude on the detection coil for all three testing elements is presented in Fig. 3, whereas the results of measurements of the tangent of phase angle between signal on measuring coil and signal applied to driving coil are shown in Fig. 4. Figures show the changes of amplitude value and of the tangent of the phase angle between signals, as a function of the rotation and linear movement of the test element.

During the tests the repeatability of measurements was verified. Standard deviation of measurements in a point doesn't exceed 1\%. Such high repeatability is very important from the point of view of accuracy of the results of further inverse tomographic transform.

\section{Conclusions}

Eddy current tomography setup presented in the paper creates possibility of tomography measurements with resolution much higher than previously reported [4]. Moreover, results presented in the paper confirm possibility of assessment of non-magnetic inclusion in ferromagnetic cylindrical elements. Value of amplitude in sensing coil changes up to $60 \%$ during the measurements, whereas tangent of angle between signal on measuring coil and signal given on driving coil changes from 0.8 to 1.35 . Repeatability of these measurements was measured by standard deviation of the value. It was about $1 \%$ for both amplitude and tangent of angle between signal on measuring coil and signal on driving coil.

As a result it was confirmed, that presented setup may be applied for non-destructive tests of cylindrical construction components without use of inconvenient X-ray radiation.

\section{References}

[1] J. Hsieh, in:Computed Tomography Principles, Design, Artifacts, and Recent Advances, Wiley, 2009.

[2] A. Tamburrino, G. Rubinacci, IEEE Transactions on Magnetics 42, 2017 (2006).

[3] D. Premel, A. Mohammad-Djafari, IEEE Transactions on Magnetics 31, 2000 (1995).

[4] M. Soleimani, Journal of Electromagnetic Waves and Applications 23, 785 (2009).

[5] M. Soleimani, A. Tamburrino, International Journal of Information and Systems Sciences 2, 343 (2006). 